\title{
On Newton-Kantorovich Method for Solving the Nonlinear Operator Equation
}

\author{
Hameed Husam Hameed, ${ }^{1,2}$ Z. K. Eshkuvatov, ${ }^{3,4}$ \\ Anvarjon Ahmedov, ${ }^{4,5}$ and N. M. A. Nik Long ${ }^{1,4}$ \\ ${ }^{1}$ Department of Mathematics, Faculty of Science, Universiti Putra Malaysia (UPM), Selangor, Malaysia \\ ${ }^{2}$ Technical Institute of Alsuwerah, The Middle Technical University, Baghdad, Iraq \\ ${ }^{3}$ Faculty of Science and Technology, Universiti Sains Islam Malaysia (USIM), Negeri Sembilan, Malaysia \\ ${ }^{4}$ Institute for Mathematical Research, Universiti Putra Malaysia (UPM), Selangor, Malaysia \\ ${ }^{5}$ Department of Process and Food Engineering, Faculty of Engineering, Universiti Putra Malaysia (UPM), Selangor, Malaysia
}

Correspondence should be addressed to Z. K. Eshkuvatov; zainidin@upm.edu.my

Received 19 July 2014; Accepted 6 October 2014

Academic Editor: Gaohang Yu

Copyright (C) 2015 Hameed Husam Hameed et al. This is an open access article distributed under the Creative Commons Attribution License, which permits unrestricted use, distribution, and reproduction in any medium, provided the original work is properly cited.

We develop the Newton-Kantorovich method to solve the system of $2 \times 2$ nonlinear Volterra integral equations where the unknown function is in logarithmic form. A new majorant function is introduced which leads to the increment of the convergence interval. The existence and uniqueness of approximate solution are proved and a numerical example is provided to show the validation of the method.

\section{Introduction}

Nonlinear phenomenon appears in many scientific areas such as physics, fluid mechanics, population models, chemical kinetics, economic systems, and medicine and can be modeled by system of nonlinear integral equations. The difficulty lies in finding the exact solution for such system. Alternatively, the approximate or numerical solutions can be sought. One of the well known approximate method is Newton-Kantorovich method which reduces the nonlinear into sequence of linear integral equations. The the approximate solution is then obtained by processing the convergent sequence. In 1939, Kantorovich [1] presented an iterative method for functional equation in Banach space and derived the convergence theorem for Newton method. In 1948, Kantorovich [2] proved a semilocal convergence theorem for Newton method in Banach space, later known as the Newton-Kantorovich method. Uko and Argyros [3] proved a weak Kantorovich-type theorem which gives the same conclusion under the weaker conditions. Shen and Li [4] have established the Kantorovich-type convergence criterion for inexact Newton methods, assuming that the first derivative of an operator satisfies the Lipschitz condition. Argyros [5] provided a sufficient condition for the semilocal convergence of Newton's method to a locally unique solution of a nonlinear operator equation. Saberi-Nadjafi and Heidari [6] introduced a combination of the Newton-Kantorovich and quadrature methods to solve the nonlinear integral equation of Urysohn type in the systematic procedure. Ezquerro et al. [7] studied the nonlinear integral equation of mixed Hammerstein type using Newton-Kantorovich method with majorant principle. Ezquerro et al. [8] provided the semilocal convergence of Newton method in Banach space under a modification of the classic conditions of Kantorovich. There are many methods of solving the system of nonlinear integral equations, for example, product integration method [9], Adomian method [10], RBF network method [11], biorthogonal system method [12], Chebyshev wavelets method [13], analytical method [14], reproducing kernel method [15], step method [16], and single term Wlash series [17]. In 2003, Boikov and Tynda [18] 
implemented the Newton-Kantorovich method to the following system:

$$
\begin{gathered}
x(t)-\int_{y(t)}^{t} h(t, \tau) g(\tau) x(\tau) d \tau=0, \\
\int_{y(t)}^{t} k(t, \tau)[1-g(\tau)] x(\tau) d \tau=f(t),
\end{gathered}
$$

where $0<t_{0} \leq t \leq T, y(t)<t$, and the functions $h(t, \tau)$, $k(t, \tau) \in C_{\left[t_{0}, T\right] \times\left[t_{0}, T\right]}, f(t), g(t) \in C_{\left[t_{0}, T\right]}$, and $(0<g(t)<1)$. In 2010, Eshkuvatov et al. [19] used the Newton-Kantorovich hypothesis to solve the system of nonlinear Volterra integral equation of the form

$$
\begin{gathered}
x(t)-\int_{y(t)}^{t} h(t, \tau) x^{2}(\tau) d \tau=0 \\
\int_{y(t)}^{t} k(t, \tau) x^{2}(\tau) d \tau=f(t)
\end{gathered}
$$

where $x(t)$ and $y(t)$ are unknown functions defined on $\left[t_{0}, \infty\right), t_{0}>0$, and $h(t, \tau), k(t, \tau) \in C_{\left[t_{0}, \infty\right] \times\left[t_{0}, \infty\right]}, f(t) \in$ $C_{\left[t_{0}, \infty\right]}$. In 2010, Eshkuvatov et al. [20] developed the modified Newton-Kantorovich to obtain an approximate solution of system with the form

$$
\begin{gathered}
x(t)-\int_{y(t)}^{t} H(t, \tau) x^{n}(\tau) d \tau=0, \\
\int_{y(t)}^{t} K(t, \tau) x^{n}(\tau) d \tau=f(t),
\end{gathered}
$$

where $0<t_{0} \leq t \leq T, y(t)<t$, and the functions $H(t$, $\tau), K(t, \tau) \in C_{\left[t_{0}, \infty\right] \times\left[t_{0}, \infty\right]}, f(t) \in C_{\left[t_{0}, \infty\right]}$, and the unknown functions $x(t) \in C_{\left[t_{0}, \infty\right]}, y(t) \in C_{\left[t_{0}, \infty\right]}^{1}, y(t)<t$.

In this paper, we consider the systems of nonlinear integral equation of the form

$$
\begin{gathered}
x(t)-\int_{y(t)}^{t} h(t, \tau) \log |x(\tau)| d \tau=g(t), \\
\int_{y(t)}^{t} k(t, \tau) \log |x(\tau)| d \tau=f(t),
\end{gathered}
$$

where $0<t_{0} \leq t \leq T, y(t)<t, x(t) \neq 0, h(t, \tau), h_{\tau}(t, \tau)$, $k(t, \tau), k_{\tau}(t, \tau) \in C(D)$ and the unknown functions $x(t) \in$ $C\left[t_{0}, T\right], y(t) \in C^{1}\left[t_{0}, T\right]$ to be determined, and $D=\left[t_{0}, T\right] \times$ $\left[t_{0}, T\right]$.

The paper is organized as follows, in Section 2, NewtonKantorovich method for the system of integral equations (4) is presented. Section 3 deals with mixed method followed by discretizations. In Section 4, the rate of convergence of the method is investigated. Lastly, Section 5 demonstrates the numerical example to verify the validity and accuracy of the proposed method, followed by the conclusion in Section 6 .

\section{Newton-Kantorovich Method for the System}

Let us rewrite the system of nonlinear Volterra integral equation (4) in the operator form

$$
P(X)=\left(P_{1}(X), P_{2}(X)\right)=0,
$$

where $X=(x(t), y(t))$ and

$$
\begin{aligned}
& P_{1}(X)=x(t)-\int_{y(t)}^{t} h(t, \tau) \log |x(\tau)| d \tau-g(t), \\
& P_{2}(X)=\int_{y(t)}^{t} k(t, \tau) \log |x(\tau)| d \tau-f(t) .
\end{aligned}
$$

To solve (5) we use initial iteration of Newton-Kantorovich method which is of the form

$$
P^{\prime}\left(X_{0}\right)\left(X-X_{0}\right)+P\left(X_{0}\right)=0
$$

where $X_{0}=\left(x_{0}(t), y_{0}(t)\right)$ is the initial guess and $x_{0}(t)$ and $y_{0}(t)$ can be any continuous functions provided that $t_{0}<$ $y(t)<t$ and $x(t) \neq 0$.

The Frechet derivative of $P(X)$ at the point $X_{0}$ is defined as

$$
\begin{gathered}
P^{\prime}\left(X_{0}\right) X \\
=\left(\lim _{s \rightarrow 0} \frac{1}{s}\left[P_{1}\left(X_{0}+s X\right)-P_{1}(X)\right],\right. \\
\left.\lim _{s \rightarrow 0} \frac{1}{s}\left[P_{2}\left(X_{0}+s X\right)-P_{2}(X)\right]\right) \\
=\left(\lim _{s \rightarrow 0} \frac{1}{s}\left[P_{1}\left(x_{0}+s x, y_{0}+s y\right)-P_{1}\left(x_{0}, y_{0}\right)\right],\right. \\
\left.\lim _{s \rightarrow 0} \frac{1}{s}\left[P_{2}\left(x_{0}+s x, y_{0}+s y\right)-P_{2}\left(x_{0}, y_{0}\right)\right]\right) \\
=\left(\operatorname { l i m } _ { s \rightarrow 0 } \left[\frac{\partial P_{1}\left(x_{0}, y_{0}\right)}{\partial x} s x+\frac{\partial P_{1}\left(x_{0}, y_{0}\right)}{\partial y} s y\right.\right. \\
+\frac{1}{2}\left(\frac{\partial^{2} P_{1}}{\partial x^{2}}\left(x_{0}+\theta s x, y_{0}+\delta s y\right) s^{2} x^{2}\right. \\
+2 \frac{\partial^{2} P_{1}}{\partial x \partial y}\left(x_{0}+\theta s x, y_{0}+\delta s y\right) s^{2} x y \\
\left.\left.+\frac{\partial^{2} P_{1}}{\partial y^{2}}\left(x_{0}+\theta s x, y_{0}+\delta s y\right) s y^{2}\right)\right]
\end{gathered}
$$




$$
\begin{gathered}
\lim _{s \rightarrow 0} \frac{1}{s}\left[\frac{\partial P_{2}}{\partial x}\left(x_{0}, y_{0}\right) s x+\frac{\partial P_{2}}{\partial y}\left(x_{0}, y_{0}\right) s y\right. \\
+\frac{1}{2}\left(\frac{\partial^{2} P_{2}}{\partial x^{2}}\left(x_{0}+\theta s x, y_{0}+\delta s y\right) s^{2} x^{2}\right. \\
+2 \frac{\partial^{2} P_{2}}{\partial x \partial y}\left(x_{0}+\theta s x, y_{0}+\delta s y\right) s^{2} x y \\
\left.\left.\left.+\frac{\partial^{2} P_{2}}{\partial y^{2}}\left(x_{0}+\theta s x, y_{0}+\delta s y\right) s y^{2}\right)\right]\right) \\
=\left(\frac{\partial P_{1}\left(x_{0}, y_{0}\right)}{\partial x} x+\frac{\partial P_{1}\left(x_{0}, y_{0}\right)}{\partial y} y,\right. \\
\left.\frac{\partial P_{2}\left(x_{0}, y_{0}\right)}{\partial x} x+\frac{\partial P_{2}\left(x_{0}, y_{0}\right)}{\partial y} y\right) .
\end{gathered}
$$

Hence,

$$
P^{\prime}\left(X_{0}\right) X=\left(\begin{array}{ll}
\left.\frac{\partial P_{1}}{\partial x}\right|_{\left(x_{0}, y_{0}\right)} & \left.\frac{\partial P_{1}}{\partial y}\right|_{\left(x_{0}, y_{0}\right)} \\
\left.\frac{\partial P_{2}}{\partial x}\right|_{\left(x_{0}, y_{0}\right)} & \left.\frac{\partial P_{2}}{\partial y}\right|_{\left(x_{0}, y_{0}\right)}
\end{array}\right)\left(\begin{array}{l}
x \\
y
\end{array}\right) .
$$

From (7) and (9) it follows that

$$
\begin{aligned}
& \left.\frac{\partial P_{1}}{\partial x}\right|_{\left(x_{0}, y_{0}\right)}(\Delta x(t))+\left.\frac{\partial P_{1}}{\partial y}\right|_{\left(x_{0}, y_{0}\right)}(\Delta y(t)) \\
& \quad=-P_{1}\left(x_{0}(t), y_{0}(t)\right), \\
& \left.\frac{\partial P_{2}}{\partial x}\right|_{\left(x_{0}, y_{0}\right)}(\Delta x(t))+\left.\frac{\partial P_{2}}{\partial y}\right|_{\left(x_{0}, y_{0}\right)}(\Delta y(t)) \\
& \quad=-P_{2}\left(x_{0}(t), y_{0}(t)\right),
\end{aligned}
$$

where $\Delta x(t)=x_{1}(t)-x_{0}(t), \Delta y(t)=y_{1}(t)-y_{0}(t)$, and $\left(x_{0}(t)\right.$, $\left.y_{0}(t)\right)$ is the initial given functions. To solve (10) with respect to $\Delta x$ and $\Delta y$ we need to compute all partial derivatives:

$$
\begin{aligned}
\left.\frac{\partial P_{1}}{\partial x}\right|_{\left(x_{0}, y_{0}\right)}= & \lim _{s \rightarrow 0} \frac{1}{s}\left(P_{1}\left(x_{0}+s x, y_{0}\right)-P_{1}\left(x_{0}, y_{0}\right)\right) \\
= & \lim _{s \rightarrow 0} \frac{1}{s}[s x(t) \\
& -\int_{y_{0}(t)}^{t} h(t, \tau)\left(\log \left|x_{0}(\tau)+s x(\tau)\right|\right. \\
= & x(t)-\int_{y_{0}(t)}^{t} h(t, \tau) \frac{x(\tau)}{x_{0}(\tau)} d \tau,
\end{aligned}
$$

$$
\begin{aligned}
\left.\frac{\partial P_{1}}{\partial y}\right|_{\left(x_{0}, y_{0}\right)} & =\lim _{s \rightarrow 0} \frac{1}{s}\left(P_{1}\left(x_{0}, y_{0}+s y\right)-P_{1}\left(x_{0}, y_{0}\right)\right) \\
& =\lim _{s \rightarrow 0} \frac{1}{s}\left[\int_{y_{0}(t)}^{y_{0}(t)+s y(t)} h(t, \tau) \log \left|\left(x_{0}(\tau)\right)\right| d \tau\right] \\
& =h\left(t, y_{0}(t)\right) \log \left|x_{0}\left(y_{0}(t)\right)\right| y(t),
\end{aligned}
$$

and in the same manner we obtain

$$
\begin{gathered}
\left.\frac{\partial P_{2}}{\partial x}\right|_{\left(x_{0}, y_{0}\right)}=\int_{y_{0}(t)}^{t} k(t, \tau) \frac{x(\tau)}{x_{0}(\tau)} d \tau, \\
\left.\frac{\partial P_{2}}{\partial y}\right|_{\left(x_{0}, y_{0}\right)}=-k\left(t, y_{0}(t)\right) \log \left|x_{0}\left(y_{0}(t)\right)\right| y(t) .
\end{gathered}
$$

So that from (10)-(12) it follows that

$$
\begin{aligned}
& \Delta x(t)-\int_{y_{0}(t)}^{t} h(t, \tau) \frac{\Delta x(\tau)}{x_{0}(\tau)} d \tau \\
&+h\left(t, y_{0}(t)\right) \log \left|x_{0}\left(y_{0}(t)\right)\right| \Delta y(t) \\
&= \int_{y_{0}(t)}^{t} h(t, \tau) \log \left|x_{0}(\tau)\right| d \tau-x_{0}(t)+g(t), \\
& \int_{y_{0}(t)}^{t} k(t, \tau) \frac{\Delta x(\tau)}{x_{0}(\tau)} d \tau \\
& \quad-k\left(t, y_{0}(t)\right) \log \left|x_{0}\left(y_{0}(t)\right)\right| \Delta y(t) \\
&=-\int_{y_{0}(t)}^{t} k(t, \tau) \log \left|x_{0}(\tau)\right| d \tau+f(t) .
\end{aligned}
$$

Equation (13) is a linear, and, by solving it for $\Delta x$ and $\Delta y$, we obtain $\left(x_{1}(t), y_{1}(t)\right)$. By continuing this process, a sequence of approximate solution $\left(x_{m}(t), y_{m}(t)\right)$ can be evaluated from

$$
P^{\prime}\left(X_{0}\right) \Delta X_{m}+P\left(X_{m}\right)=0
$$

which is equivalent to the system

$$
\begin{aligned}
& \Delta x_{m}(t)-\int_{y_{0}(t)}^{t} h(t, \tau) \frac{\Delta x_{m}(\tau)}{x_{0}(\tau)} d \tau \\
& \quad+h\left(t, y_{0}(t)\right) \log \left|x_{0}\left(y_{0}(t)\right)\right| \Delta y_{m}(t) \\
& =\int_{y_{0}(t)}^{t} h(t, \tau) \log \left|x_{0}(\tau)\right| d \tau-x_{0}(t)+g(t), \\
& \int_{y_{0}(t)}^{t} k(t, \tau) \frac{\Delta x_{m}(\tau)}{x_{0}(\tau)} d \tau \\
& \quad-k\left(t, y_{0}(t)\right) \log \left|x_{0}\left(y_{0}(t)\right)\right| \Delta y_{m}(t) \\
& =-\int_{y_{0}(t)}^{t} k(t, \tau) \log \left|x_{0}(\tau)\right| d \tau+f(t),
\end{aligned}
$$

where $\Delta x_{m}(t)=x_{m}(t)-x_{m-1}(t)$ and $\Delta y_{m}(t)=y_{m}(t)-y_{m-1}(t)$, $m=1,2,3, \ldots$. 
Thus, one should solve a system of two linear Volterra integral equations to find each successive approximation. Let us eliminate $\Delta y(t)$ from the system (13) by finding the expression of $\Delta y(t)$ from the first equation of this system and substitute it in the second equation to yield

$$
\begin{aligned}
& \Delta y(t)=\frac{1}{H(t)}\left[\int_{y_{0}(t)}^{t} h(t, \tau)\left[\frac{\Delta x(\tau)}{x_{0}(\tau)}+\log \left|x_{0}(\tau)\right|\right] d \tau\right. \\
& \left.-\left[\Delta x(t)+x_{0}(t)-g(t)\right]\right], \\
& G(t)\left[\int_{y_{0}(t)}^{t} h(t, \tau)\left[\frac{\Delta x(\tau)}{x_{0}(\tau)}+\log \left|x_{0}(\tau)\right|\right] d \tau\right. \\
& \left.-\left[\Delta x(t)+x_{0}(t)-g(t)\right]\right] \\
& =\int_{y_{0}(t)}^{t} k(t, \tau) \frac{\Delta x(\tau)}{x_{0}(\tau)} d \tau \\
& -\int_{y_{0}(t)}^{t} k(t, \tau) \log \left|x_{0}(\tau)\right| d \tau+f(t),
\end{aligned}
$$

where $G(t)=k\left(t, y_{0}(t)\right) / h\left(t, y_{0}(t)\right)$ and $H(t)=1 /[h(t$, $\left.\left.y_{0}(t)\right) \log \left|x_{0}\left(y_{0}(t)\right)\right|\right]$, and the second equation of (16) yields

$$
\Delta x(t)-\int_{y_{0}(t)}^{t} k_{1}(t, \tau) \frac{\Delta x(\tau)}{x_{0}(\tau)} \tau=F_{0}(t),
$$

where

$$
\begin{gathered}
k_{1}(t, \tau)=h(t, \tau)-\frac{k(t, \tau)}{G(t)}, \\
G(t)=\frac{k\left(t, y_{0}(t)\right)}{h\left(t, y_{0}(t)\right)}, \quad k\left(t, y_{0}(t)\right) \neq 0 \forall t \in\left[t_{0}, T\right], \\
F_{0}(t)=\int_{y_{0}(t)}^{t} k_{1}(t, \tau) \log \left|x_{0}(\tau)\right| d \tau-x_{0}(t)+g(t)+\frac{f(t)}{G(t)} .
\end{gathered}
$$

In an analogous way, $\Delta y_{m}(t)$ and $\Delta x_{m}(t)$ can be written in the form

$$
\begin{aligned}
& \Delta y_{m}(t) \\
& =\frac{1}{H(t)}\left[\int_{y_{0}(t)}^{t} h(t, \tau) \frac{\Delta x_{m}(\tau)}{x_{0}(\tau)} d \tau\right. \\
& \quad+\int_{y_{m-1}(t)}^{t} h(t, \tau) \log \left|x_{m-1}(\tau)\right| d \tau \\
& \left.\quad-\Delta x_{m}(t)-x_{m-1}(t)+g(t)\right], \\
& \Delta x_{m}(t)-\int_{y_{0}(t)}^{t} k_{1}(t, \tau) \frac{\Delta x_{m}(\tau)}{x_{0}(\tau)} d \tau=F_{m-1}(t),
\end{aligned}
$$

where

$$
\begin{aligned}
F_{m-1}(t)= & \int_{y_{m-1}(t)}^{t} k_{1}(t, \tau) \log \left|x_{m-1}(\tau)\right| d \tau-x_{m-1}(t) \\
& +g(t)+\frac{f(t)}{G(t)} .
\end{aligned}
$$

\section{The Mixed Method (Simpson and Trapezoidal) for Approximate Solution}

At each step of the iterative process we have to find the solution of (18) and (20) on the closed interval $\left[t_{0}, T\right]$. To do this the grid $(\omega)$ of points $t_{i}=t_{0}+i h, i=1,2,3, \ldots, 2 N$, $h=\left(T-t_{0}\right) / 2 N$ is introduced, and by the collocation method with mixed rule we require that the approximate solution satisfies (18) and (20). Hence

$$
\begin{gathered}
\Delta x_{m}\left(t_{0}\right)=-x_{m-1}\left(t_{0}\right)+g\left(t_{0}\right)+\frac{f\left(t_{0}\right)}{G\left(t_{0}\right)}, \\
\Delta x_{m}\left(t_{2 i}\right)-\int_{y_{0}\left(t_{2 i}\right)}^{t_{2 i}} k_{1}\left(t_{2 i}, \tau\right) \frac{\Delta x_{m}(\tau)}{x_{0}(\tau)} d \tau \\
=F_{m-1}\left(t_{2 i}\right), \quad i=1,2, \ldots, N .
\end{gathered}
$$

On the grid $(\omega)$ we set $v_{2 i}=y_{0}\left(t_{2 i}\right)$, suct that

$$
t_{v_{2 i}}= \begin{cases}t_{v_{2 i}}, & t_{0} \leq y_{0}\left(t_{2 i}\right)<t_{2 i-2} \\ t_{2 i}, & t_{2 i-2} \leq y_{0}\left(t_{2 i}\right)<t_{2 i}\end{cases}
$$

Consequently, the system (23) can be written in the form

$$
\begin{gathered}
\Delta x_{m}\left(t_{2 i}\right)-\int_{y_{0}\left(t_{2 i}\right)}^{t_{v_{2 i}}} k_{1}\left(t_{2 i}, \tau\right) \frac{\Delta x_{m}(\tau)}{x_{0}(\tau)} d \tau \\
\quad-\sum_{j=v_{2 i}}^{i-1} \int_{t_{2 j}}^{t_{2 j+2}} k_{1}\left(t_{2 i}, \tau\right) \frac{\Delta x_{m}(\tau)}{x_{0}(\tau)} d \tau \\
=F_{m-1}\left(t_{2 i}\right), \quad i=1,2, \ldots, N .
\end{gathered}
$$

By computing the integral in (26) using tapezoidal formula on the first integrals and Simpson formula on the second integral, we consider two cases.

Case 1 . When $v_{2 i} \neq 2 i, i=1,2, \ldots, N$, then

$$
\Delta x_{m}\left(t_{2 i}\right)=\frac{F_{m-1}\left(t_{2 i}\right)+A(i)+B(i)+C(i)}{1-\left(\left(t_{2 i}-t_{2 i-2}\right) / 6 \cdot x_{0}\left(t_{2 i}\right)\right) k_{1}\left(t_{2 i}, t_{2 i}\right)},
$$


where

$$
\begin{aligned}
& A(i)=0.5\left(t_{v_{2 i}}-y_{0}\left(t_{2 i}\right)\right) \\
& \times\left[k_{1}\left(t_{2 i}, t_{v_{2 i}}\right) \frac{\Delta x_{m}\left(t_{v_{2 i}}\right)}{x_{0}\left(t_{v_{2 i}}\right)}+k_{1}\left(t_{2 i}, y_{0}\left(t_{2 i}\right)\right)\right. \\
& \times \frac{\Delta x_{m}\left(t_{v_{2 i}}\right)\left(t_{v_{2 i}}-y_{0}\left(t_{2 i}\right)\right)}{\left(t_{v_{2 i}}-t_{v_{2 i-2}}\right)\left(x_{0}\left(y_{0}\left(t_{2 i}\right)\right)\right)} \\
& +k_{1}\left(t_{2 i}, y_{0}\left(t_{2 i}\right)\right) \\
& \left.\times \frac{\Delta x_{m}\left(t_{v_{2 i-2}}\right)\left(y_{0}\left(t_{2 i}\right)-t_{v_{2 i-2}}\right)}{\left(t_{v_{2 i}}-t_{v_{2 i-2}}\right)\left(x_{0}\left(y_{0}\left(t_{2 i}\right)\right)\right)}\right], \\
& B(i)=\sum_{j=v_{2 i}}^{i-2} \frac{\left(t_{2 j+2}-t_{2 j}\right)}{6} \\
& \times\left[k_{1}\left(t_{2 i}, t_{2 j}\right) \frac{\Delta x_{m}\left(t_{2 j}\right)}{x_{0}\left(t_{2 j}\right)}\right. \\
& +4 k_{1}\left(t_{2 i}, t_{2 j+1}\right) \frac{\Delta x_{m}\left(t_{2 j+1}\right)}{x_{0}\left(t_{2 j+1}\right)} \\
& \left.+k_{1}\left(t_{2 i}, t_{2 j+2}\right) \frac{\Delta x_{m}\left(t_{2 j+2}\right)}{x_{0}\left(t_{2 j+2}\right)}\right] \text {, } \\
& C(i)=\frac{\left(t_{2 i}-t_{2 i-2}\right)}{6}\left[k_{1}\left(t_{2 i}, t_{2 i-2}\right) \frac{\Delta x_{m}\left(t_{2 i-2}\right)}{x_{0}\left(t_{2 i-2}\right)}\right. \\
& \left.+4 k_{1}\left(t_{2 i}, t_{2 i-1}\right) \frac{\Delta x_{m}\left(t_{2 i-1}\right)}{x_{0}\left(t_{2 i-1}\right)}\right] \text {. }
\end{aligned}
$$

Case 2. When $v_{2 i}=2 i, i=1,2, \ldots, N$, then

$$
\Delta x_{m}\left(t_{2 i}\right)=\frac{D_{1}(i)}{D_{2}(i)}
$$

where

$$
\begin{aligned}
D_{1}(i)= & F_{m-1}\left(t_{2 i}\right)+0.5 k_{1}\left(t_{2 i}, y_{0}\left(t_{2 i}\right)\right) \\
\times & {\left[\frac{\Delta x_{m}\left(t_{2 i-2}\right)}{x_{0}\left(y_{0}\left(t_{2 i}\right)\right)} \frac{\left(t_{2 i}-y_{0}\left(t_{2 i}\right)\right)\left(y_{0}\left(t_{2 i}\right)-t_{2 i-2}\right)}{t_{2 i}-t_{2 i-2}}\right], } \\
D_{2}(i)= & {\left[1-0.5\left(t_{2 i}-y_{0}\left(t_{2}\right)\right) \frac{k_{1}\left(t_{2 i}, t_{2 i}\right)}{x_{0}\left(t_{2 i}\right)}\right.} \\
& \left.-0.5 k_{1}\left(t_{2 i}, y_{0}\left(t_{2 i}\right)\right) \frac{\left(t_{2 i}-y_{0}\left(t_{2 i}\right)\right)^{2}}{x_{0}\left(y_{0}\left(t_{2 i}\right)\right)\left(t_{2 i}-t_{2 i-2}\right)}\right] .
\end{aligned}
$$

Also, to compute $\Delta y_{m}(t)$ on the grid $(\omega)$, (18) can be represented in the form

$$
\begin{aligned}
\Delta y_{m}\left(t_{2 i}\right)= & \frac{1}{H\left(t_{2 i}\right)} \\
& \times\left[\int_{y_{0}\left(t_{2 i}\right)}^{t_{2 i}} h\left(t_{2 i}, \tau\right) \frac{\Delta x_{m}(\tau)}{x_{0}(\tau)} d \tau\right. \\
& \quad+\int_{y_{m-1}\left(t_{2 i}\right)}^{t_{2 i}} h\left(t_{2 i}, \tau\right) \log \left|x_{m-1}(\tau)\right| d \tau \\
& \left.\quad-\Delta x_{m}\left(t_{2 i}\right)-x_{m-1}\left(t_{2 i}\right)+g\left(t_{2 i}\right)\right] .
\end{aligned}
$$

Let us set $v_{2 i}=y_{0}\left(t_{2 i}\right)$ and $u_{2 i}=y_{m-1}\left(t_{2 i}\right)$ and

$$
\begin{gathered}
t_{v_{2 i}}= \begin{cases}t_{2 i}, & t_{2 i-2} \leq y_{0}\left(t_{2 i}\right)<t_{2 i}, \\
t_{v_{2 i}}, & t_{0} \leq y_{0}\left(t_{2 i}\right)<t_{2 i-2},\end{cases} \\
t_{u_{2 i}}= \begin{cases}t_{2 i}, & t_{2 i-2} \leq y_{m-1}\left(t_{2 i}\right)<t_{2 i}, \\
t_{u_{2 i}}, & t_{0} \leq y_{m-1}\left(t_{2 i}\right)<t_{2 i-2} .\end{cases}
\end{gathered}
$$

Then (30) can be written as

$$
\begin{aligned}
\Delta y_{m}\left(t_{2 i}\right)=\frac{1}{H\left(t_{2 i}\right)} & \\
& \times\left[\int_{y_{0}\left(t_{2 i}\right)}^{t_{v_{2 i}}} h\left(t_{2 i}, \tau\right) \frac{\Delta x_{m}(\tau)}{x_{0}(\tau)} d \tau\right. \\
& +\sum_{j=v_{2 i}}^{i-1} \int_{t_{2 j}}^{t_{2 j+2}} h\left(t_{2 i}, \tau\right) \frac{\Delta x_{m}(\tau)}{x_{0}(\tau)} d \tau \\
& +\int_{y_{m-1}\left(t_{2 i}\right)}^{t_{u_{2 i}}} h\left(t_{2 i}, \tau\right) \log \left|x_{m-1}(\tau)\right| d \tau \\
& +\sum_{j=u_{2 i}}^{i-1} \int_{t_{2 j}}^{t_{2 j+2}} h\left(t_{2 i}, \tau\right) \log \left|x_{m-1}(\tau)\right| d \tau \\
& \left.\quad-\Delta x_{m}\left(t_{2 i}\right)-x_{m-1}\left(t_{2 i}\right)+g\left(t_{2 i}\right)\right]
\end{aligned}
$$

and by applying mixed formula for (32) we obtain the following four cases. 
Case 1 . When $v_{2 i} \neq 2 i$ and $u_{2 i} \neq 2 i$, we have

$$
\begin{aligned}
& \Delta y_{m}\left(t_{2 i}\right) \\
& =\frac{1}{H\left(t_{2 i}\right)} \\
& \times\left[0.5\left(t_{v_{2 i}}-y_{0}\left(t_{2 i}\right)\right)\right. \\
& \times\left(h\left(t_{2 i}, t_{v_{2 i}}\right) \frac{\Delta x_{m}\left(t_{v_{2 i}}\right)}{x_{0}\left(t_{v_{2 i}}\right)}\right. \\
& \left.+h\left(t_{2 i}, y_{0}\left(t_{2 i}\right)\right) \frac{\Delta x_{m}\left(y_{0}\left(t_{2 i}\right)\right)}{x_{0}\left(y_{0}\left(t_{2 i}\right)\right)}\right) \\
& +\sum_{j=v_{2 i}}^{i-1} \frac{\left(t_{2 j+2}-t_{2 j}\right)}{6} \\
& \times\left(h\left(t_{2 i}, t_{2 j}\right) \frac{\Delta x_{m}\left(t_{2 j}\right)}{x_{0}\left(t_{2 j}\right)}\right. \\
& +4 h\left(t_{2 i}, t_{2 j+1}\right) \frac{\Delta x_{m}\left(t_{2 j+1}\right)}{x_{0}\left(t_{2 j+1}\right)} \\
& \left.+h\left(t_{2 i}, t_{2 j+2}\right) \frac{\Delta x_{m}\left(t_{2 j+2}\right)}{x_{0}\left(t_{2 j+2}\right)}\right) \\
& +0.5\left(t_{u_{2 i}}-y_{m-1}\left(t_{2 i}\right)\right) \\
& \times\left(h\left(t_{2 i}, t_{u_{2 i}}\right) \log \left|x_{m-1}\left(t_{u_{2 i}}\right)\right|\right. \\
& \left.+h\left(t_{2 i}, y_{m-1}\left(t_{2 i}\right)\right) \log \left|\left(x_{m-1}\left(y_{m-1}\left(t_{2 i}\right)\right)\right)\right|\right) \\
& +\sum_{j=u_{2 i}}^{i-1} \frac{\left(t_{2 j+2}-t_{2 j}\right)}{6} \\
& \times\left(h\left(t_{2 i}, t_{2 j}\right) \log \left(x_{m-1}\left(t_{2 j}\right)\right)\right. \\
& +4 h\left(t_{2 i}, t_{2 j+1}\right) \log \left|x_{m-1}\left(t_{2 j+1}\right)\right| \\
& \left.+h\left(t_{2 i}, t_{2 j+2}\right) \log \left|x_{m-1}\left(t_{2 j+2}\right)\right|\right) \\
& \left.-\Delta x_{m}\left(t_{2 i}\right)-x_{m-1}\left(t_{2 i}\right)+g\left(t_{2 i}\right)\right] \text {. }
\end{aligned}
$$

Case 2. If $v_{2 i}=2 i$ and $u_{2 i} \neq 2 i$, then

$$
\begin{aligned}
& \Delta y_{m}\left(t_{2 i}\right) \\
& =\frac{1}{H\left(t_{2 i}\right)} \\
& \times\left[0.5\left(t_{2 i}-y_{0}\left(t_{2 i}\right)\right)\right. \\
& \times\left(h\left(t_{2 i}, t_{2 i}\right) \frac{\Delta x_{m}\left(t_{v_{2 i}}\right)}{x_{0}\left(t_{2 i}\right)}\right. \\
& \left.+h\left(t_{2 i}, y_{0}\left(t_{2 i}\right)\right) \frac{\Delta x_{m}\left(y_{0}\left(t_{2 i}\right)\right)}{x_{0}\left(y_{0}\left(t_{2 i}\right)\right)}\right) \\
& +0.5\left(t_{u_{2 i}}-y_{m-1}\left(t_{2 i}\right)\right) \\
& \times\left(h\left(t_{2 i}, t_{u_{2 i}}\right) \log \left|x_{m-1}\left(t_{u_{2 i}}\right)\right|\right. \\
& \left.+h\left(t_{2 i}, y_{m-1}\left(t_{2 i}\right)\right) \log \left|x_{m-1}\left(y_{m-1}\left(t_{2 i}\right)\right)\right|\right) \\
& +\sum_{j=u_{2 i}}^{i-1} \frac{\left(t_{2 j+2}-t_{2 j}\right)}{6} \\
& \times\left(h\left(t_{2 i}, t_{2 j}\right) \log \left|x_{m-1}\left(t_{2 j}\right)\right|\right. \\
& +4 h\left(t_{2 i}, t_{2 j+1}\right) \log \left|x_{m-1}\left(t_{2 j+1}\right)\right| \\
& \left.+h\left(t_{2 i}, t_{2 j+2}\right) \log \left|x_{m-1}\left(t_{2 j+2}\right)\right|\right) \\
& \left.-\Delta x_{m}\left(t_{2 i}\right)-x_{m-1}\left(t_{2 i}\right)+g\left(t_{2 i}\right)\right] \text {. }
\end{aligned}
$$

Case 3. When $v_{2 i} \neq 2 i$ and $u_{2 i}=2 i$, we get

$$
\begin{aligned}
& \Delta y_{m}\left(t_{2 i}\right) \\
& =\frac{1}{H\left(t_{2 i}\right)} \\
& \times \quad\left[\begin{array}{l}
0.5\left(t_{v_{2 i}}-y_{0}\left(t_{2 i}\right)\right) \\
\quad \times\left(h\left(t_{2 i}, t_{v_{2 i}}\right) \frac{\Delta x_{m}\left(t_{v_{2 i}}\right)}{x_{0}\left(t_{v_{2 i}}\right)}\right. \\
\left.\quad+h\left(t_{2 i}, y_{0}\left(t_{2 i}\right)\right) \frac{\Delta x_{m}\left(y_{0}\left(t_{2 i}\right)\right)}{x_{0}\left(y_{0}\left(t_{2 i}\right)\right)}\right)
\end{array}\right.
\end{aligned}
$$




$$
\begin{aligned}
& +\sum_{j=v_{2 i}}^{i-1} \frac{\left(t_{2 j+2}-t_{2 j}\right)}{6} \\
& \times\left(h\left(t_{2 i}, t_{2 j}\right) \frac{\Delta x_{m}\left(t_{2 j}\right)}{x_{0}\left(t_{2 j}\right)}\right. \\
& +4 h\left(t_{2 i}, t_{2 j+1}\right) \frac{\Delta x_{m}\left(t_{2 j+1}\right)}{x_{0}\left(t_{2 j+1}\right)} \\
& \left.+h\left(t_{2 i}, t_{2 j+2}\right) \frac{\Delta x_{m}\left(t_{2 j+2}\right)}{x_{0}\left(t_{2 j+2}\right)}\right) \\
& +0.5\left(t_{2 i}-y_{m-1}\left(t_{2 i}\right)\right) \\
& \times\left(h\left(t_{2 i}, t_{2 i}\right) \log \left|x_{m-1}\left(t_{2 i}\right)\right|\right. \\
& \left.+h\left(t_{2 i}, y_{m-1}\left(t_{2 i}\right)\right) \log \left|x_{m-1}\left(y_{m-1}\left(t_{2 i}\right)\right)\right|\right) \\
& \left.-\Delta x_{m}\left(t_{2 i}\right)-x_{m-1}\left(t_{2 i}\right)+g\left(t_{2 i}\right)\right] .
\end{aligned}
$$

Case 4. If $v_{2 i}=2 i$ and $u_{2 i}=2 i$, then

$$
\begin{aligned}
& \Delta y_{m}\left(t_{2 i}\right) \\
& =\frac{1}{H\left(t_{2 i}\right)} \\
& \times\left[0.5\left(t_{2 i}-y_{0}\left(t_{2 i}\right)\right)\right. \\
& \times\left(h\left(t_{2 i}, t_{2 i}\right) \frac{\Delta x_{m}\left(t_{2 i}\right)}{x_{0}\left(t_{2 i}\right)}\right. \\
& \left.+h\left(t_{2 i}, y_{0}\left(t_{2 i}\right)\right) \frac{\Delta x_{m}\left(y_{0}\left(t_{2 i}\right)\right)}{x_{0}\left(y_{0}\left(t_{2 i}\right)\right)}\right) \\
& +0.5\left(t_{2 i}-y_{m-1}\left(t_{2 i}\right)\right) \\
& \times\left(h\left(t_{2 i}, t_{2 i}\right) \log \left|x_{m-1}\left(t_{2 i}\right)\right|\right. \\
& \left.+h\left(t_{2 i}, y_{m-1}\left(t_{2 i}\right)\right) \log \left|x_{m-1}\left(y_{m-1}\left(t_{2 i}\right)\right)\right|\right) \\
& \left.-\Delta x_{m}\left(t_{2 i}\right)-x_{m-1}\left(t_{2 i}\right)+g\left(t_{2 i}\right)\right] \text {. }
\end{aligned}
$$

Thus, (32) can be computed by one of (33)-(36) according to the cases.

\section{The Convergence Analysis of the Method}

On the basis of general theorems of Newton-Kantorovich method [21, Chapter XVIII] for the convergence, we state the following theorem regarding the successive approximations described by (18)-(20).

First, consider the following classes of functions:

(i) $C_{\left[t_{0}, T\right]}$ the set of all continuous functions $f(t)$ defined on the interval $\left[t_{0}, T\right]$,

(ii) $C_{\left[t_{0}, T\right] \times\left[t_{0}, T\right]}$ the set of all continuous functions $\psi(t, \tau)$ defined on the region $\left[t_{0}, T\right] \times\left[t_{0}, T\right]$,

(iii) $\bar{C}=\left\{X: X=(x(t), y(t)): x(t), y(t) \in C_{\left[t_{0}, T\right]}\right\}$,

(iv) $C_{\left[t_{0}, T\right]}^{<}=\left\{y(t) \in C_{\left[t_{0}, T\right]}^{1}: y(t)<t\right\}$.

And define the following norms

$$
\begin{aligned}
& \|x\|=\max _{t \in\left[t_{0}, T\right]}|x(t)|, \\
& \|\Delta X\|_{\bar{C}}=\max \left\{\|\Delta x\|_{C_{\left[t_{0}, T\right]}},\|\Delta y\|_{C_{\left[t_{0}, T\right]}}\right\} \text {, } \\
& \|X\|_{C^{1}}=\max \left\{\|x\|_{C_{\left[t_{0}, T\right]}},\left\|x^{\prime}\right\|_{C_{\left[t_{0}, T\right]}}\right\} \text {, } \\
& \|\bar{X}\|_{\bar{C}}=\max \left\{\|\bar{x}\|_{C_{\left[t_{0}, T\right]}},\|\bar{y}\|_{C_{\left[t_{0}, T\right]}}\right\} \\
& \|h(t, \tau)\|=H_{1}, \quad\left\|h_{\tau}^{\prime}(t, \tau)\right\|=H_{1}^{\prime}, \\
& \|k(t, \tau)\|=H_{2}, \quad\left\|k_{\tau}^{\prime}(t, \tau)\right\|=H_{2}^{\prime}, \\
& \left\|\frac{1}{x_{0}}\right\|=\max _{t \in\left[t_{0}, T\right]}\left|\frac{1}{x_{0}(t)}\right|=c_{1} \text {, } \\
& \left\|\frac{1}{x_{0}^{2}}\right\|=\max _{t \in\left[t_{0}, T\right]}\left|\frac{1}{x_{0}^{2}(t)}\right|=c_{2} \text {, } \\
& \left\|\frac{1}{G(t)}\right\|=\max _{t \in\left[t_{0}, T\right]}\left|\frac{1}{G(t)}\right|=c_{3}, \\
& \left\|x_{0}\right\|=\max _{t \in\left[t_{0}, T\right]}\left|x_{0}(t)\right|=H_{3}, \\
& \left\|x_{0}^{\prime}\right\|=\max _{t \in\left[t_{0}, T\right]}\left|x_{0}^{\prime}(t)\right|=H_{3}^{\prime} \text {, } \\
& \min _{t \in\left[t_{0}, T\right]}\left|y_{0}(t)\right|=H_{4}, \\
& \|\log \|=\max _{t \in\left[t_{0}, T\right]}|\log (x(t))|=H_{5}, \\
& \|g\|=\max _{t \in\left[t_{0}, T\right]}|g(t)|=H_{6} \text {, } \\
& \|f\|=\max _{t \in\left[t_{0}, T\right]}|f(t)|=H_{7} .
\end{aligned}
$$

Let

$$
\begin{array}{r}
\eta_{1}=\max \left\{H_{1} c_{2}\left(T-H_{4}\right), H_{1} c_{1}, H_{1}^{\prime} H_{5}+H_{1} H_{3}^{\prime} c_{1},\right. \\
\left.H_{2} c_{2}\left(T-H_{4}\right), H_{2} c_{1}, H_{2}^{\prime} H_{5}+H_{2} H_{3}^{\prime} c_{1}\right\} .
\end{array}
$$


Let us consider real valued function

$$
\psi(t)=K\left(t-t_{0}\right)^{2}-(1+K \eta)\left(t-t_{0}\right)+\eta
$$

where $K>0$ and $\eta$ are nonnegative real coefficients.

Theorem 1. Assume that the operator $P(X)=0$ in (5) is defined in $\Omega=\left\{X \in C\left(\left[t_{0}, T\right]\right):\left\|X-X_{0}\right\| \leq R\right\}$ and has continuous second derivative in closed ball $\Omega_{0}=\left\{X \in C\left(\left[t_{0}\right.\right.\right.$, $\left.T]):\left\|X-X_{0}\right\| \leq r\right\}$ where $T=t_{0}+r \leq t_{0}+R$. Suppose the following conditions are satisfied:

(1) $\left\|\Gamma_{0} P\left(X_{0}\right)\right\| \leq \eta /(1+K \eta)$,

(2) $\left\|\Gamma_{0} P^{\prime \prime}(X)\right\| \leq 2 K /(1+K \eta)$, when $\left\|X-X_{0}\right\| \leq t-t_{0} \leq r$,

where $K$ and $\eta$ as in (39). Then the function $\psi(t)$ defined by (39) majorizes the operator $P(X)$.

Proof. Let us rewrite (5) and (39) in the form

$$
\begin{gathered}
t=\phi(t), \quad \phi(t)=t+c_{0} \psi(t), \\
X=S(X), \quad S(X)=X-\Gamma_{0} P(X),
\end{gathered}
$$

where $c_{0}=-1 / \psi^{\prime}\left(t_{0}\right)=1 /(1+K \eta)$ and $\Gamma_{0}=\left[P^{\prime}\left(X_{0}\right)\right]^{-1}$.

Let us show that (40) and (41) satisfy the majorizing conditions [21, Theorem 1, page 525]. In fact

$$
\left\|S\left(X_{0}\right)-X_{0}\right\|=\left\|-\Gamma_{0} P\left(X_{0}\right)\right\| \leq \frac{\eta}{1+K \eta}=\phi\left(t_{0}\right)-t_{0},
$$

and for the $\left\|X-X_{0}\right\| \leq t-t_{0}$ with the Remark in [21, Remark 1 , page 504] we have

$$
\begin{aligned}
\left\|S^{\prime}(X)\right\| & =\left\|S^{\prime}(X)-S^{\prime}\left(X_{0}\right)\right\| \\
& \leq \int_{X_{0}}^{X}\left\|S^{\prime \prime}(X)\right\| d X=\int_{X_{0}}^{X}\left\|\Gamma_{0} P^{\prime \prime}(X)\right\| d X \\
& \leq \int_{t_{0}}^{t} c_{0} \psi^{\prime \prime}(\tau) d \tau=\int_{t_{0}}^{t} \frac{2 K}{1+K \eta} d \tau \\
& =\frac{2 K}{1+K \eta}\left(t-t_{0}\right)=\phi^{\prime}(t) .
\end{aligned}
$$

Hence $\psi(t)=0$ is a majorant function of $P(X)=0$.

Theorem 2. Let the functions $f(t), g(t) \in C_{\left[t_{0}, T\right]}, x_{0}(t) \in C^{1}\left[t_{0}\right.$, $T], x_{0}\left(y_{0}(t)\right) \neq 0, x_{0}^{2}(t) \neq 0$, and the kernels $h(t, \tau), k(t, \tau) \in$ $C_{\left[t_{0}, T\right] \times\left[t_{0}, T\right]}^{1}$ and $\left(x_{0}(t), y_{0}(t)\right) \in \Omega_{0}$; then

(1) the system (7) has unique solution in the interval $\left[t_{0}\right.$, $T]$; that is, there exists $\Gamma_{0}$, and $\left\|\Gamma_{0}\right\| \leq \sum_{j=1}^{\infty}\left(c_{1} H_{1}+\right.$ $\left.c_{1} c_{3} H_{2}\right)^{j}\left(\left(T-H_{4}\right)^{j-1} /(j-1) !\right)=\eta_{2}$,

(2) $\|\Delta X\| \leq \eta /(1+K \eta)$,

(3) $\left\|P^{\prime \prime}(X)\right\| \leq \eta_{1}$,

(4) $\eta>1 / K$ and $r<\eta+t_{0}$, where $K$ and $\eta$ as in (39). Then the system (4) has unique solution $X^{*}$ in the closed ball $\Omega_{0}$ and the sequence $X_{m}(t)=$ $\left(x_{m}(t), y_{m}(t)\right), m \geq 0$ of successive approximations

$$
\begin{aligned}
& \Delta y_{m}(t)=\frac{1}{H(t)}\left[\int_{y_{0}(t)}^{t} h(t, \tau) \frac{\Delta x_{m}(\tau)}{x_{0}(\tau)} d \tau\right. \\
&+\int_{y_{m-1}(t)}^{t} h(t, \tau) \log \left|x_{m-1}(\tau)\right| d \tau \\
&\left.-\Delta x_{m}(t)-x_{m-1}(t)+g(t)\right], \\
& \Delta x_{m}(t)-\int_{y_{0}(t)}^{t} k_{1}(t, \tau) \frac{\Delta x_{m}(\tau)}{x_{0}(\tau)} d \tau=F_{m-1}(t),
\end{aligned}
$$

where $\Delta x_{m}(t)=x_{m}(t)-x_{m-1}(t)$ and $\Delta y_{m}(t)=y_{m}(t)-y_{m-1}(t)$, $m=2,3, \ldots$, and $X_{m}$ converge to the solution $X^{*}$. The rate of convergence is given by

$$
\left\|X^{*}-X_{m}\right\| \leq\left(\frac{2}{1+K \eta}\right)^{m}\left(\frac{1}{K}\right) .
$$

Proof. It is shown that (7) is reduced to (17). Since (17) is a linear Volterra integral equation of 2 nd kind with respect to $\Delta x(t)$ and since $k\left(t, y_{0}(t)\right) \neq 0, \forall t \in\left[t_{0}, T\right]$ which implies that the kernel $k_{1}(t, \tau)$ defined by (18) is continues it follows that (17) has a unique solution which can be obtained by the method of successive approximations. Then the function $\Delta y(t)$ is uniquely determined from (16). Hence the existence of $\Gamma_{0}$ is archived.

To verify that $\Gamma_{0}$ is bounded we need to establish the resolvent kernel $\Gamma_{0}(t, \tau)$ of (17), so we assume the integral operator $U$ from $C\left[t_{0}, T\right] \rightarrow C\left[t_{0}, T\right]$ is given by

$$
Z=U(\Delta x), \quad Z(t)=\int_{y_{0}(t)}^{t} k_{2}(t, \tau) \Delta x(\tau) d \tau,
$$

where $k_{2}(t, \tau)=k_{1}(t, \tau) / x_{0}(\tau)$, and $k_{1}(t, \tau)$ is defined in (18).

Due to (46), (17) can be written as

$$
\Delta x-U(\Delta x)=F_{0} .
$$

The solution $\Delta x^{*}$ of (47) is expressed in terms of $F_{0}$ by means of the formula

$$
\Delta x^{*}=F_{0}+B\left(F_{0}\right) \text {, }
$$

where $B$ is an integral operator and can be expanded as a series in powers of $U$ [21, Theorem 1, page 378]:

$$
B\left(F_{0}\right)=U\left(F_{0}\right)+U^{2}\left(F_{0}\right)+\cdots+U^{n}\left(F_{0}\right)+\cdots,
$$

and it is known that the powers of $U$ are also integral operators. In fact

$$
\begin{array}{r}
Z_{n}=U^{n}, \quad Z_{n}(t)=\int_{y_{0}(t)}^{t} k_{2}^{(n)}(t, \tau) \Delta x(\tau) d \tau, \\
(n=1,2, \ldots),
\end{array}
$$

where $k_{2}^{(n)}$ is the iterated kernel. 
Substituting (50) into (48) we obtain an expression for the solution of (47):

$$
\Delta x^{*}=F_{0}(t)+\sum_{j=1}^{\infty} \int_{y_{0}(t)}^{t} k_{2}^{(j)}(t, \tau) F_{0}(\tau) d \tau .
$$

Next, we show that the series in (51) is convergent uniformly for all $t \in\left[t_{0}, T\right]$. Since

$$
\begin{aligned}
\left|k_{2}(t, \tau)\right| & =\left|\frac{k_{1}(t, \tau)}{x_{0}(\tau)}\right| \\
& \leq\left|\frac{h(t, \tau)}{x_{0}(\tau)}\right|+\left|\frac{k(t, \tau)}{x_{0}(\tau) G(t)}\right| \leq c_{1} H_{1}+c_{1} c_{3} H_{2} .
\end{aligned}
$$

Let $M=c_{1} H_{1}+c_{1} c_{3} H_{2}$; then by mathematical induction we get

$$
\begin{aligned}
\left|k_{2}^{(2)}(t, \tau)\right| & \leq \int_{y_{0}(t)}^{t}\left|k_{2}(t, u) k_{2}(u, \tau)\right| d u \leq \frac{M^{2}\left(t-H_{4}\right)}{(1) !}, \\
\left|k_{2}^{(3)}(t, \tau)\right| & \leq \int_{y_{0}(t)}^{t}\left|k_{2}(t, u) k_{2}^{(2)}(u, \tau)\right| d u \leq \frac{M^{3}\left(t-H_{4}\right)^{2}}{(2) !}, \\
& \vdots \\
\left|k_{2}^{(n)}(t, \tau)\right| & \leq \int_{y_{0}(t)}^{t}\left|k_{2}(t, u) k_{2}^{(n-1)}(u, \tau)\right| d u \\
& \leq \frac{M^{n}\left(t-H_{4}\right)^{n-1}}{(n-1) !} \quad \quad(n=1,2, \ldots) ;
\end{aligned}
$$

then

$$
\left\|U^{n}\right\|=\max _{t \in\left[t_{0}, T\right]} \int_{y_{0}(t)}^{t}\left|k_{2}^{(n)}(t, \tau)\right| d \tau \leq \frac{M^{n}\left(T-H_{4}\right)^{(n-1)}}{(n-1) !} .
$$

Therefore the $n$th root test of the sequence yields

$$
\sqrt[n]{\left\|U^{n}\right\|} \leq \frac{M\left(T-H_{4}\right)^{1-1 / n}}{\sqrt[n]{(n-1) !}} \underset{n \rightarrow \infty}{\longrightarrow} 0 .
$$

Hence $\rho=1 / \lim _{n \rightarrow \infty} \sqrt[n]{\left\|U^{n}\right\|}=\infty$ and a Volterra integral equations (17) has no characteristic values. Since the series in (51) converges uniformly (48) can be written in terms of resolvent kernel of (17):

$$
\Delta x^{*}=F_{0}+\int_{y_{0}(t)}^{t} \Gamma_{0}(t, \tau) F_{0}(\tau) d \tau,
$$

where

$$
\Gamma_{0}(t, \tau)=\sum_{j=1}^{\infty} k_{2}^{(j)}(t, \tau)
$$

Since the series in (57) is convergent we obtain

$$
\left\|\Gamma_{0}\right\|=\left\|B\left(F_{0}\right)\right\| \leq \sum_{j=1}^{\infty}\left\|U^{j}\right\| \leq \sum_{j=1}^{\infty} M^{j} \frac{(T-H)^{j-1}}{(j-1) !} \leq \eta_{2} .
$$

To establish the validity of second condition, let us represent operator equation

$$
P(X)=0
$$

as in (41) and its the successive approximations is

$$
X_{n+1}=S\left(X_{n}\right), \quad(n=0,1,2, \ldots) .
$$

For initial guess $X_{0}$ we have

$$
S\left(X_{0}\right)=X_{0}-\Gamma_{0} P\left(X_{0}\right)
$$

From second condition of (Theorem 1) we have

$$
\begin{aligned}
\left\|\Gamma_{0} P\left(X_{0}\right)\right\| & =\left\|S\left(X_{0}\right)-X_{0}\right\| \\
& =\left\|X_{1}-X_{0}\right\|=\|\Delta X\| \leq \frac{\eta}{1+K \eta} .
\end{aligned}
$$

In addition, we need to show that $\left\|P^{\prime \prime}(X)\right\| \leq \eta_{1}$ for all $X \in \Omega_{0}$ where $\eta_{1}$ is defined in (38). It is known that the second derivative $P^{\prime \prime}\left(X_{0}\right)(X, \bar{X})$ of the nonlinear operator $P(X)$ is described by 3-dimensional array $P^{\prime \prime}\left(X_{0}\right) X \bar{X}=$ $\left(D_{1}, D_{2}\right)(X, \bar{X})$, which is called bilinear operator; that is, $P^{\prime \prime}\left(X_{0}\right)(X \bar{X})=B\left(X_{0}, X, \bar{X}\right)$ where

$$
\begin{aligned}
& P^{\prime \prime}\left(X_{0}\right)(X, \bar{X}) \\
&=\lim _{s \rightarrow 0} \frac{1}{s}\left[P^{\prime}\left(x_{0}+s \bar{X}\right)-P^{\prime}\left(X_{0}\right)\right] \\
&=\left\{\operatorname { l i m } _ { s \rightarrow 0 } \frac { 1 } { s } \left[\left(\frac{\partial P_{1}}{\partial x}\left(x_{0}+s \bar{x}, y_{0}+s \bar{y}\right)-\frac{\partial P_{1}}{\partial x}\left(x_{0}, y_{0}\right)\right) x\right.\right. \\
&\left.+\left(\frac{\partial P_{1}}{\partial y}\left(x_{0}+s \bar{x}, y_{0}+s \bar{y}\right)-\frac{\partial P_{1}}{\partial y}\left(x_{0}, y_{0}\right)\right) y\right], \\
& \lim _{s \rightarrow 0} \frac{1}{s}\left[\left(\frac{\partial P_{2}}{\partial x}\left(x_{0}+s \bar{x}, y_{0}+s \bar{y}\right)-\frac{\partial P_{2}}{\partial x}\left(x_{0}, y_{0}\right)\right) x\right. \\
&\left.\left.\quad+\left(\frac{\partial P_{2}}{\partial y}\left(x_{0}+s \bar{x}, y_{0}+s \bar{y}\right)-\frac{\partial P_{2}}{\partial y}\left(x_{0}, y_{0}\right)\right) y\right]\right\}
\end{aligned}
$$




$$
\begin{aligned}
& =\left\{\operatorname { l i m } _ { s \rightarrow 0 } \frac { 1 } { s } \left[\left(\frac{\partial^{2} P_{1}}{\partial x^{2}}\left(x_{0}, y_{0}\right) s \bar{x}+\frac{\partial^{2} P_{1}}{\partial y \partial x}\left(x_{0}, y_{0}\right) s \bar{y}\right.\right.\right. \\
& +\frac{1}{2}\left(\frac{\partial^{3} P_{1}}{\partial x^{3}}\left(x_{0}+\theta s \bar{x}, y_{0}+\delta s \bar{y}\right) s^{2} \bar{x}^{2}\right. \\
& +2 \frac{\partial^{3} P_{1}}{\partial x^{2} \partial y}\left(x_{0}+\theta s \bar{x}, y_{0}+\delta s \bar{y}\right) s^{2} \bar{x} \bar{y} \\
& \left.\left.+\frac{\partial^{3} P_{1}}{\partial y^{2} \partial x}\left(x_{0}+\theta s \bar{x}, y_{0}+\delta s \bar{y}\right) s^{2} \bar{y}\right)\right) x \\
& +\left(\frac{\partial^{2} P_{1}}{\partial x \partial y}\left(x_{0}, y_{0}\right) s \bar{x}+\frac{\partial^{2} P_{1}}{\partial y^{2}}\left(x_{0}, y_{0}\right) s \bar{y}\right. \\
& +\frac{1}{2}\left(\frac{\partial^{3} P_{1}}{\partial x^{2} \partial y}\left(x_{0}+\theta s \bar{x}, y_{0}+\delta s \bar{y}\right) s^{2} \bar{x}^{2}\right. \\
& +2 \frac{\partial^{3} P_{1}}{\partial x \partial y^{2}}\left(x_{0}+\theta s \bar{x}, y_{0}+\delta s \bar{y}\right) s^{2} \bar{x} \bar{y} \\
& \left.\left.\left.+\frac{\partial^{3} P_{1}}{\partial y^{3}}\left(x_{0}+\theta s \bar{x}, \delta s \bar{y}\right) s^{2} \bar{y}^{2}\right)\right) y\right] \\
& \lim _{s \rightarrow 0} \frac{1}{s}\left[\left(\frac{\partial^{2} P_{2}}{\partial x^{2}}\left(x_{0}, y_{0}\right) s \bar{x}+\frac{\partial^{2} P_{2}}{\partial y \partial x}\left(x_{0}, y_{0}\right) s \bar{y}\right.\right.
\end{aligned}
$$$$
+\frac{1}{2}\left(\frac{\partial^{3} P_{2}}{\partial x^{3}}\left(x_{0}+\theta s \bar{x}, y_{0}+\delta s \bar{y}\right) s^{2} \bar{x}^{2}\right.
$$$$
+2 \frac{\partial^{3} P_{2}}{\partial x^{2} \partial y}\left(x_{0}+\theta s \bar{x}, y_{0}+\delta s \bar{y}\right) s^{2} \bar{x} \bar{y}
$$$$
\left.\left.+\frac{\partial^{3} P_{2}}{\partial y^{2} \partial x}\left(x_{0}+\theta s \bar{x}, y_{0}+\delta s \bar{y}\right) s^{2} \bar{y}\right)\right) x
$$$$
+\left(\frac{\partial^{2} P_{2}}{\partial x \partial y}\left(x_{0}, y_{0}\right) s \bar{x}+\frac{\partial^{2} P_{2}}{\partial y^{2}}\left(x_{0}, y_{0}\right) s \bar{y}\right.
$$$$
+\frac{1}{2}\left(\frac{\partial^{3} P_{2}}{\partial x^{2} \partial y}\left(x_{0}+\theta s \bar{x}, y_{0}+\delta s \bar{y}\right) s^{2} \bar{x}^{2}\right.
$$$$
+2 \frac{\partial^{3} P_{2}}{\partial x \partial y^{2}}\left(x_{0}+\theta s \bar{x}, y_{0}+\delta s \bar{y}\right) s^{2} \bar{x} \bar{y}
$$$$
\left.\left.\left.\left.+\frac{\partial^{3} P_{2}}{\partial y^{3}}\left(x_{0}+\theta s \bar{x}, \delta s \bar{y}\right) s^{2} \bar{y}^{2}\right)\right) y\right]\right\}
$$$$
=\left(\frac{\partial^{2} P_{1}}{\partial x^{2}}\left(x_{0}, y_{0}\right) \bar{x} x+\frac{\partial^{2} P_{1}}{\partial y \partial x}\left(x_{0}, y_{0}\right) \bar{y} x\right.
$$$$
+\frac{\partial^{2} P_{1}}{\partial x \partial y}\left(x_{0}, y_{0}\right) \bar{x} y+\frac{\partial^{2} P_{1}}{\partial y^{2}}\left(x_{0}, y_{0}\right) \bar{y} x,
$$$$
\frac{\partial^{2} P_{2}}{\partial x^{2}}\left(x_{0}, y_{0}\right) \bar{x} x+\frac{\partial^{2} P_{2}}{\partial y \partial x}\left(x_{0}, y_{0}\right) \bar{y} x
$$$$
\left.+\frac{\partial^{2} P_{2}}{\partial x \partial y}\left(x_{0}, y_{0}\right) \bar{x} y+\frac{\partial^{2} P_{2}}{\partial y^{2}}\left(x_{0}, y_{0}\right) \bar{y} x\right) \text {, }
$$

where $\theta, \delta \in(0,1)$, so we have

$$
P^{\prime \prime}\left(X_{0}\right)(X, \bar{X})=\left(\begin{array}{ll}
D_{1} & D_{2}
\end{array}\right)\left(\begin{array}{l}
\bar{x} \\
\bar{y}
\end{array}\right)\left(\begin{array}{l}
x \\
y
\end{array}\right),
$$

where

$$
\begin{gathered}
D_{1}=\left(\begin{array}{cc}
\left.\frac{\partial^{2} P_{1}}{\partial x^{2}}\right|_{\left(x_{0}, y_{0}\right)} & \left.\frac{\partial^{2} P_{1}}{\partial y \partial x}\right|_{\left(x_{0}, y_{0}\right)} \\
\left.\frac{\partial^{2} P_{1}}{\partial x \partial y}\right|_{\left(x_{0}, y_{0}\right)} & \left.\frac{\partial^{2} P_{1}}{\partial y^{2}}\right|_{\left(x_{0}, y_{0}\right)}
\end{array}\right), \\
D_{2}=\left(\begin{array}{cc}
\left.\frac{\partial^{2} P_{2}}{\partial x^{2}}\right|_{\left(x_{0}, y_{0}\right)} & \left.\frac{\partial^{2} P_{2}}{\partial y \partial x}\right|_{\left(x_{0}, y_{0}\right)} \\
\left.\frac{\partial^{2} P_{2}}{\partial x \partial y}\right|_{\left(x_{0}, y_{0}\right)} & \left.\frac{\partial^{2} P_{2}}{\partial y^{2}}\right|_{\left(x_{0}, y_{0}\right)}
\end{array}\right) .
\end{gathered}
$$

Then the norms of every components of $D_{1}$ and $D_{2}$ have the estimate

$$
\begin{aligned}
& \left\|\frac{\partial^{2} P_{1}}{\partial x^{2}}\right\|=\max _{\|X\| \leq 1,\|\bar{X}\| \leq 1}\left|\int_{y_{0}(t)}^{t} h(t, \tau) \frac{x(\tau)}{x_{0}^{2}(\tau)} \bar{x}(\tau) d \tau\right| \\
& \leq H_{1} c_{2}\left(T-H_{4}\right), \\
& \left\|\frac{\partial^{2} P_{1}}{\partial x \partial y}\right\|=\max _{\|X\| \leq 1,\|\bar{X}\| \leq 1}\left|h\left(t, y_{0}(t)\right) \frac{x\left(y_{0}(t)\right)}{x_{0}\left(y_{0}(t)\right)} \bar{y}(t)\right| \leq H_{1} c_{1} \text {, } \\
& \left\|\frac{\partial^{2} P_{1}}{\partial y \partial x}\right\|=\max _{\|X\| \leq 1,\|\bar{X}\| \leq 1}\left|h\left(t, y_{0}(t)\right) \frac{\bar{x}\left(y_{0}(t)\right)}{x_{0}\left(y_{0}(t)\right)} y(t)\right| \leq H_{1} c_{1}, \\
& \left\|\frac{\partial^{2} P_{1}}{\partial y^{2}}\right\|=\max _{\|X\| \leq 1,\|\bar{X}\| \leq 1} \mid\left[h_{\tau}^{\prime}\left(t, y_{0}(t)\right) \log \left|x_{0}\left(y_{0}(t)\right)\right|\right. \\
& \left.+h\left(t, y_{0}(t)\right) \frac{x_{0}^{\prime}\left(y_{0}(t)\right)}{x_{0}\left(y_{0}(t)\right)}\right] \\
& \times y(t) \bar{y}(t) \\
& \leq H_{1}^{\prime} H_{5}+H_{1} H_{3}^{\prime} c_{1}, \\
& \left\|\frac{\partial^{2} P_{2}}{\partial x^{2}}\right\|=\max _{\|X\| \leq 1,\|\bar{X}\| \leq 1}\left|\int_{y_{0}(t)}^{t} k(t, \tau) \frac{x(\tau)}{x_{0}^{2}(\tau)} \bar{x}(\tau) d \tau\right| \\
& \leq H_{2} c_{2}\left(T-H_{4}\right) \text {, } \\
& \left\|\frac{\partial^{2} P_{2}}{\partial x \partial y}\right\|=\max _{\|X\| \leq 1,\|\bar{X}\| \leq 1}\left|-k\left(t, y_{0}(t)\right) \frac{x\left(y_{0}(t)\right)}{x_{0}\left(y_{0}(t)\right)} \bar{y}(t)\right| \\
& \leq H_{2} c_{1} \text {, } \\
& \left\|\frac{\partial^{2} P_{2}}{\partial y \partial x}\right\|=\max _{\|X\| \leq 1,\|\bar{X}\| \leq 1}\left|-k\left(t, y_{0}(t)\right) \frac{\bar{x}\left(y_{0}(t)\right)}{x_{0}\left(y_{0}(t)\right)} y(t)\right| \\
& \leq H_{2} c_{1} \text {, }
\end{aligned}
$$




$$
\begin{aligned}
& \left\|\frac{\partial^{2} P_{2}}{\partial y^{2}}\right\|=\max _{\|X\| \leq 1,\|\bar{X}\| \leq 1} \mid\left[k_{\tau}^{\prime}\left(t, y_{0}(t)\right) \log \left|x_{0}\left(y_{0}(t)\right)\right|\right. \\
& \left.+k\left(t, y_{0}(t)\right) \frac{x_{0}^{\prime}\left(y_{0}(t)\right)}{x_{0}\left(y_{0}(t)\right)}\right] \\
& \times y(t) \bar{y}(t) \mid \\
& \leq H_{2}^{\prime} H_{5}+H_{2} H_{3}^{\prime} c_{1} .
\end{aligned}
$$

Therefore, all the second derivatives exist and are bounded:

$$
\left\|P^{\prime \prime}(X)\right\| \leq \eta_{1} \text {. }
$$

Since $\psi(t)$ majorizes operator $P(X)$ and utilizing the second condition of (Theorem 1 ) we get

$$
\left\|\Gamma_{0} P^{\prime \prime}(X)\right\| \leq \frac{2 K}{1+K \eta} .
$$

Let us consider the discriminant of equation $\psi(t)=0$ :

$$
D=K^{2} \eta^{2}-2 K \eta+1=(k \eta-1)^{2},
$$

and the two roots of $\psi(t)=0$ are $r_{1}=1 / K+t_{0}$ and $r_{2}=\eta+t_{0}$; therefore, when $r_{1}<r<r_{2}$ implies

$$
\psi(r) \leq 0
$$

then under the assumption of the fourth condition, that is, $1 / K+t_{0}$ is the unique solution of $\psi(t)=0$ in $\left[t_{0}, T\right]$ and the condition in (70) [21, Theorem 4, page 530] implies that $X^{*}$ is the unique solution of operator equation (5) [21, Theorem 6, page 532] and

$$
\left\|X^{*}-X_{0}\right\| \leq t^{*}-t_{0}
$$

where $t^{*}$ is the unique solution of $\psi(t)=0$ in $\left[t_{0}, r\right]$.

To show the rate of convergence let us write the equation $\psi(t)=0$ in a same form as in (40) then its successive approximation is

$$
t_{m+1}=\phi\left(t_{m}\right), \quad m=0,1,2, \ldots
$$

To estimate the difference between $t^{*}$ and successive approximation $t_{m}$ :

$$
t^{*}-t_{m}=\phi\left(t^{*}\right)-\phi\left(t_{m-1}\right)=\phi^{\prime}\left(\widetilde{t_{m}}\right)\left(t^{*}-t_{m-1}\right),
$$

where $\widetilde{t_{m}} \in\left(t_{m-1}, t^{*}\right)$ and

$$
\phi^{\prime}(t)=1+c_{0} \psi^{\prime}(t)=\frac{2 K}{1+K \eta}\left(t-t_{0}\right) ;
$$

therefore

$$
\begin{aligned}
\phi^{\prime}\left(\widetilde{t_{m}}\right) & =\frac{2 K}{1+K \eta}\left(\widetilde{t_{m}}-t_{0}\right) \\
& \leq \frac{2 K}{1+K \eta}\left(t^{*}-t_{0}\right)=\frac{2}{1+K \eta} ;
\end{aligned}
$$

then

$$
\begin{aligned}
t^{*}-t_{m} & \leq \frac{2}{1+K \eta}\left(t^{*}-t_{m-1}\right), \\
t^{*}-t_{m-1} & \leq \frac{2}{1+K \eta}\left(t^{*}-t_{m-2}\right) ; \\
& \vdots \\
t^{*}-t_{1} & \leq \frac{2}{1+K \eta}\left(t^{*}-t_{0}\right),
\end{aligned}
$$

consequently,

$$
t^{*}-t_{m} \leq\left(\frac{2}{1+K \eta}\right)^{m} \frac{1}{K}
$$

it implies

$$
\left\|X^{*}-X_{m}\right\| \leq\left(t^{*}-t_{m}\right) \leq\left(\frac{2}{1+K \eta}\right)^{m} \frac{1}{K} .
$$

\section{Numerical Example}

Consider the system of nonlinear equation

$$
\begin{gathered}
x(t)-\int_{y(t)}^{t} t \tau \log (|x(\tau)|) d \tau=e^{t}-\frac{t^{2}}{3}, \\
\int_{y(t)}^{t} \tau \log (|x(\tau)|) d \tau=\frac{t}{3}, \quad t \in[10,15] .
\end{gathered}
$$

The exact solution is

$$
\begin{gathered}
x^{*}(t)=e^{t}, \\
y^{*}(t)=\sqrt[3]{t^{3}-t},
\end{gathered}
$$

and the initial guesses are

$$
\begin{gathered}
x_{0}(t)=e^{10}(t-9), \\
y_{0}(t)=0.6 t+4 .
\end{gathered}
$$

Table 1 shows that $x_{m}(t)$ coincides with the exact $x^{*}(t)$ from the first iteration whereas only six iterations are needed for $y_{m}(t)$ to be very close to $y^{*}(t)$. Notations used here are as follows: $N$ is the number of nodes, $m$ is the number of iterations, and $\epsilon_{x}=\max _{t \in[10,15]}\left|x_{m}(t)-x^{*}(t)\right|$ and $\epsilon_{y}=$ $\max _{t \in[10,15]}\left|y_{m}(t)-y^{*}(t)\right|$.

\section{Conclusion}

In this paper, the Newton-Kantorovich method is developed to solve the system of nonlinear Volterra integral equations which contains logarithmic function. We have introduced a new majorant function that leads to the increment of range of convergence of successive approximation process. A new theorem is stated based on the general theorems of Kantorovich. Numerical example is given to show the validation of the method. Table 1 shows that the proposed method is in good agreement with the theoretical findings. 
TABLE 1: Numerical results for (79).

\begin{tabular}{lcc}
\hline & $N=20, h=0.25$ \\
$m$ & $\epsilon_{x}$ & $\epsilon_{y}$ \\
\hline 1 & 0.00 & 0.0029 \\
2 & 0.00 & $4.3597 E-006$ \\
3 & 0.00 & $3.1061 E-008$ \\
4 & 0.00 & $1.0140 E-009$ \\
5 & 0.00 & $1.2541 E-010$ \\
6 & 0.00 & $3.9968 E-011$ \\
\hline
\end{tabular}

\section{Conflict of Interests}

The authors declare that there is no conflict of interests regarding the publication of this paper.

\section{Acknowledgment}

This work was supported by University Putra Malaysia under Fundamental Research Grant Scheme (FRGS). Project code is $01-12-10-989 \mathrm{FR}$.

\section{References}

[1] L. V. Kantorovich, "The method of successive approximation for functional equations," Acta Mathematica, vol. 71, no. 1, pp. 6397, 1939.

[2] L. V. Kantorovich, “On Newton's method for functional equations," Doklady Akademii Nauk SSSR, vol. 59, pp. 1237-1240, 1948 (Russian).

[3] L. U. Uko and I. K. Argyros, "A weak Kantorovich existence theorem for the solution of nonlinear equations," Journal of Mathematical Analysis and Applications, vol. 342, no. 2, pp. 909914, 2008.

[4] W. Shen and C. Li, "Kantorovich-type convergence criterion for inexact Newton methods," Applied Numerical Mathematics, vol. 59, no. 7, pp. 1599-1611, 2009.

[5] I. K. Argyros, “On Newton's method for solving equations containing Fréchet-differentiable operators of order at least two," Applied Mathematics and Computation, vol. 215, no. 4, pp. 1553-1560, 2009.

[6] J. Saberi-Nadjafi and M. Heidari, "Solving nonlinear integral equations in the Urysohn form by Newton-Kantorovichquadrature method," Computers \& Mathematics with Applications, vol. 60, no. 7, pp. 2058-2065, 2010.

[7] J. A. Ezquerro, D. González, and M. A. Hernández, "A variant of the Newton-Kantorovich theorem for nonlinear integral equations of mixed Hammerstein type," Applied Mathematics and Computation, vol. 218, no. 18, pp. 9536-9546, 2012.

[8] J. A. Ezquerro, D. González, and M. A. Hernández, "A modification of the classic conditions of Newton-Kantorovich for Newton's method," Mathematical and Computer Modelling, vol. 57, no. 3-4, pp. 584-594, 2013.

[9] B. Jumarhon and S. McKee, "Product integration methods for solving a system of nonlinear Volterra integral equations," Journal of Computational and Applied Mathematics, vol. 69, no. 2, pp. 285-301, 1996.

[10] H. Sadeghi Goghary, S. Javadi, and E. Babolian, "Restarted Adomian method for system of nonlinear Volterra integral equations," Applied Mathematics and Computation, vol. 161, no. 3, pp. 745-751, 2005.

[11] A. Golbabai, M. Mammadov, and S. Seifollahi, "Solving a system of nonlinear integral equations by an RBF network," Computers \& Mathematics with Applications, vol. 57, no. 10, pp. 1651-1658, 2009.

[12] M. I. Berenguer, D. Gámez, A. I. Garralda-Guillem, M. Ruiz Galán, and M. C. Serrano Pérez, "Biorthogonal systems for solving Volterra integral equation systems of the second kind," Journal of Computational and Applied Mathematics, vol. 235, no. 7, pp. 1875-1883, 2011.

[13] J. Biazar and H. Ebrahimi, "Chebyshev wavelets approach for nonlinear systems of Volterra integral equations," Computers \& Mathematics with Applications, vol. 63, no. 3, pp. 608-616, 2012.

[14] M. Ghasemi, M. Fardi, and R. Khoshsiar Ghaziani, "Solution of system of the mixed Volterra-Fredholm integral equations by an analytical method," Mathematical and Computer Modelling, vol. 58, no. 7-8, pp. 1522-1530, 2013.

[15] L.-H. Yang, H.-Y. Li, and J.-R. Wang, "Solving a system of linear Volterra integral equations using the modified reproducing kernel method," Abstract and Applied Analysis, vol. 2013, Article ID 196308, 5 pages, 2013.

[16] M. Dobritoiu and M.-A. Serban, "Step method for a system of integral equations from biomathematics," Applied Mathematics and Computation, vol. 227, pp. 412-421, 2014.

[17] V. Balakumar and K. Murugesan, "Single-term Walsh series method for systems of linear Volterra integral equations of the second kind," Applied Mathematics and Computation, vol. 228, pp. 371-376, 2014.

[18] I. V. Boikov and A. N. Tynda, "Approximate solution of nonlinear integral equations of the theory of developing systems," Differential Equations, vol. 39, no. 9, pp. 1277-1288, 2003.

[19] Z. K. Eshkuvatov, A. Ahmedov, N. M. A. Nik Long, and O. Shafiq, "Approximate solution of the system of nonlinear integral equation by Newton-Kantorovich method," Applied Mathematics and Computation, vol. 217, no. 8, pp. 3717-3725, 2010.

[20] Z. K. Eshkuvatov, A. Ahmedov, N. M. A. Nik Long, and O. Shafiq, "Approximate solution of nonlinear system of integral equation using modified Newton-Kantorovich method," in Proceeding of the Reggional Annual Fumdamental Science Seminar, pp. 595-607, 2010.

[21] L. V. Kantorovich and G. P. Akilov, functional Analysis, Pergamon Press, Nauka, 1982. 


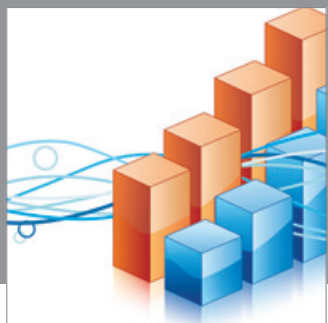

Advances in

Operations Research

mansans

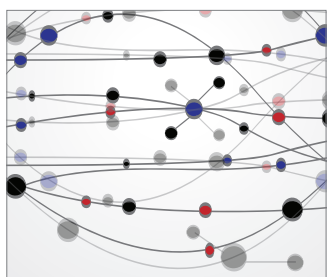

The Scientific World Journal
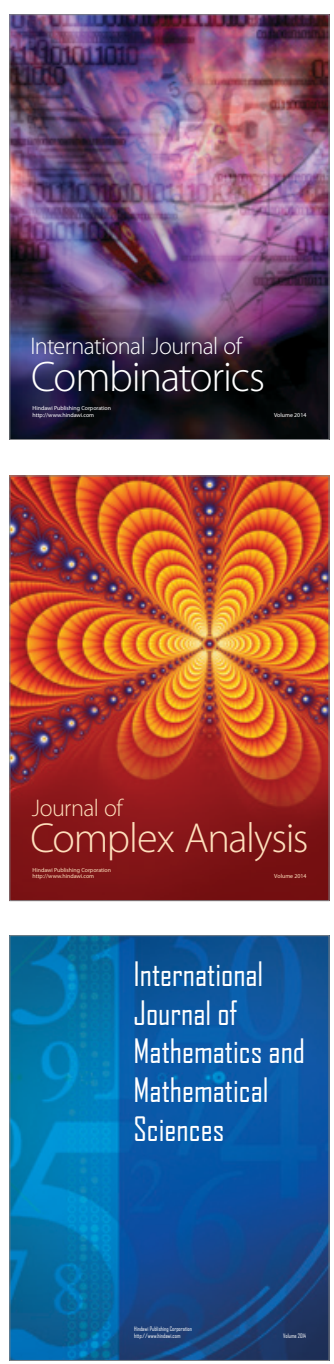
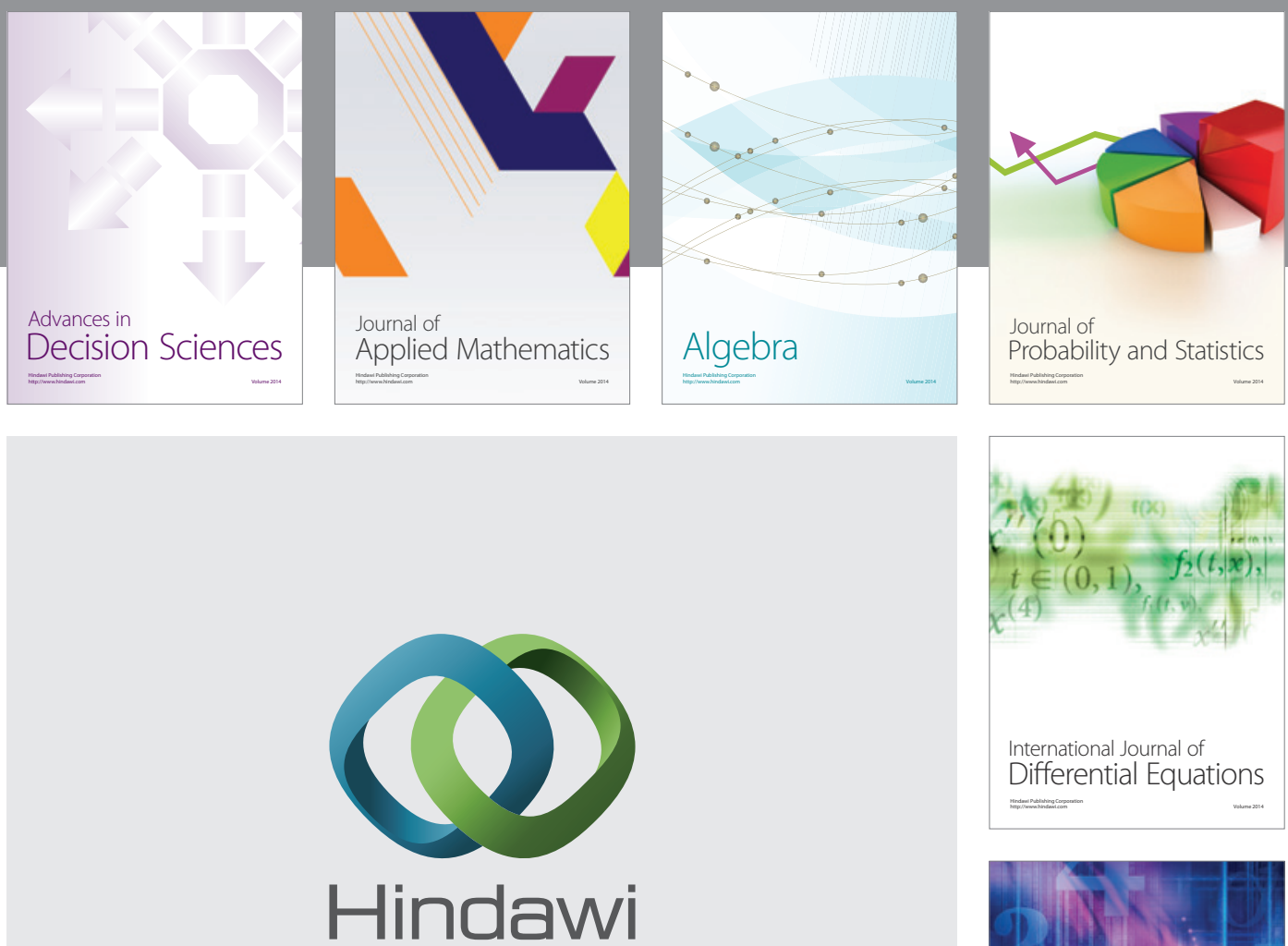

Submit your manuscripts at http://www.hindawi.com
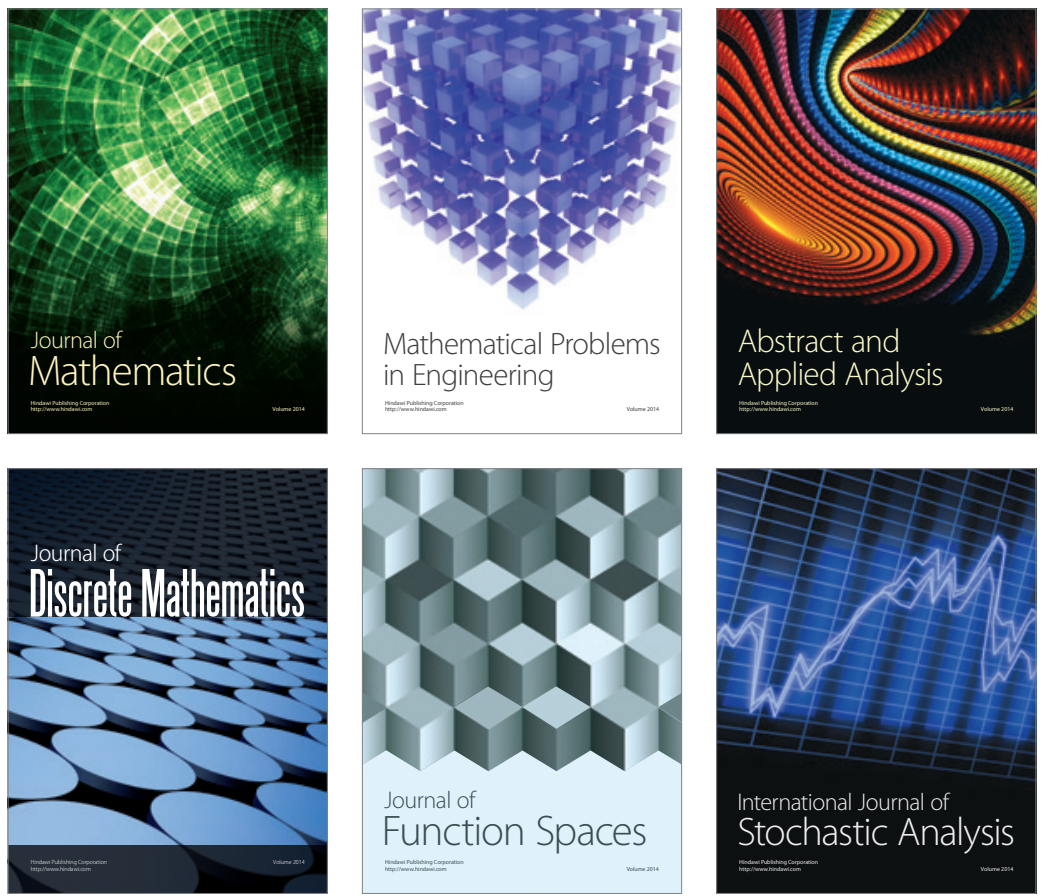

Journal of

Function Spaces

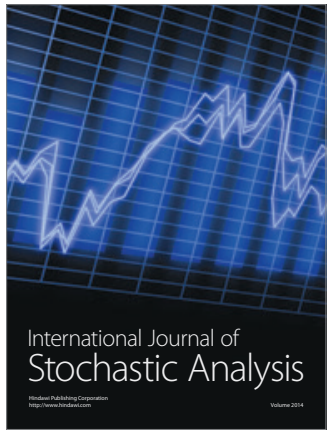

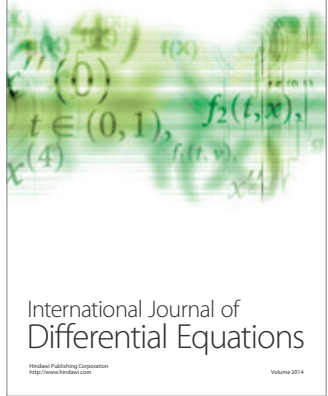
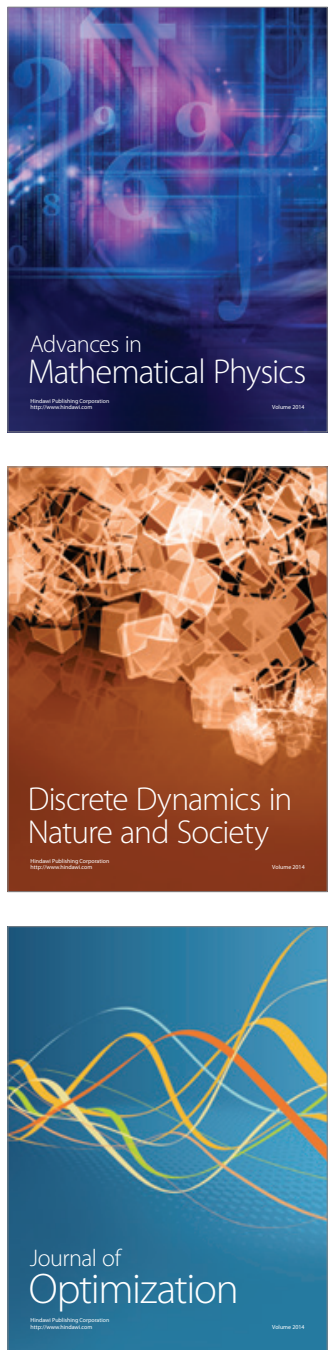